\title{
THE ANALYSIS OF THE THREE-LEVEL HM-NETWORK AND ITS APPLICATION FOR THE INCOMES FORECASTING OF A LOGISTIC TRANSPORT SYSTEM
}

\author{
Mikhail Matalytski ${ }^{1}$, Olga Kiturko ${ }^{2}$ \\ ${ }^{I}$ Institute of Mathematics, Czestochowa University of Technology \\ Częstochowa, Poland \\ ${ }^{2}$ Faculty of Mathematics and Computer Science, Grodno State University \\ Grodno, Belarus \\ Im.matalytski@gmail.com, ${ }^{2}$ sytaya_om@mail.ru
}

\begin{abstract}
Investigation of the closed three-level HM-network, which is the model of the production transportation in a logistic transport system, is conducted in this article. The approximated expression for the expected income of the central system and the system of the nonhomogeneous differential equations for an average number of messages in network systems, when the incomes from transitions between network's states are random variables with the set moments of the first two orders, is received. The relation for variance of the income of the central system of network (system of the top level) is also received.
\end{abstract}

Keywords: HM-network, a logistic transport system, expected incomes, variance of the income

\section{Introduction}

The methods of making decisions in the conditions of uncertainty while optimizing the logistic systems (LS) are more and more in demand [1]. In solving such problems we have to deal with the situations when finding of the optimum decision becomes more complicated because of the need of the simultaneous accounting of a set of the random factors whose probabilistic characteristics aren't often known in advance.

In article [2] the procedures of minimization of total expenses at the service of portfolios of orders in the links of chains of deliveries, in case of the arbitrary laws of distribution of a period of their service, are presented. In [3] it is shown that the accounting of a decrease in tariffs for expenses in time doesn't change the optimum strategy. It is proved that the specified feature always takes place at the exponential and the geometrical laws of distribution of periods of the service of orders. It is specified that tariffs for expenses in reality can be time functions, and depend on how long the order expects the beginning of the service. In [4] the probabilistic model of system of the supply consisting of several plants, warehouses and 
the consumers, allowing one to predict financial risks is developed and to take optimum decisions.

The above allows one to make a conclusion that the probabilistic methods for the solution of theoretical and practical tasks in logistics are usually used nowadays. Random transport flows between various subjects of the logistic transport systems (LTS), flows of the production arriving from producers to recipients in random time periods, random duration of time intervals that are necessary for production, loading and unloading, storage in warehouses and sales, random volumes of transported freights and the spaces occupied by them in the warehouse, predetermined the need to use the methods of the queueing theory (QT) for development of the mathematical models applied in logistics. In [5] the application of the Markov queueing systems (QS) for determination of number of an integrated team of transport and warehouse workers for freight loading according to the technological scheme «a warehouse - a loader - the car» is described. The importance of the application of the Markov QS as adequate models of functioning of LTS is noted in [6].

It is clear that the queueing network can be used to describe a functioning of various producers, warehouses and consumers of some LS as one system, where various queueing systems of the network correspond to different subjects of LS. For LTS the tasks of an assessment and forecasting of income of their subjects, gained from transportation of production by different types of transport between subjects, are important. Let's assume that we enter $n$ subjects $S_{1}, S_{2}, \ldots, S_{n}$ into the described LTS (it can be manufacturing plants, warehouses, consumers) between which transportation of products is carried out. Production transportation by car from one subject to another brings it some certain income gained of realized production, and the income of the other subject decreases by some size. Thus expenses on transportation (fuel, car repairs), the salary of the driver can be carried both to the first and to the second subject. Times of unloading-loading of cars in subjects of LTS and streams of cars between them are casual. It is necessary to estimate (to predict) the expected (average) income of subjects of LTS from such transportations. The similar situation arises at an assessment and the forecasting of the income of the transport enterprise (TE) which is carrying out transportation of products between subjects. Freight transportation from the subject $S_{i}$ to the subject $S_{j}$ brings to TE some, in a general case random incomes, but at the same time TE has a loss of property. For the solution of such problems in this article HM-networks with time-dependent parameters are used.

\section{Markov networks with the incomes}

It is known that functioning of any Markov queueing network is described by means of Markov's chains with continuous time. In work [7] R. Howard introduced the concept of Markov chains with the constant incomes. For the analysis of such 
chains with a small number of states he offered using a method of transformations of Laplace and a method of one-dimensional z-transformations. This concept was laid down in a basis for definition of the Markov queueing networks with the income which were entered for the first time into consideration in work [8]. The closed networks with a large number of states at the investigation of which there is a problem of dimension $[9,10]$ or in general open networks with calculating number of states $[11,12]$ were considered. And in the beginning the investigation was conducted of the closed and open networks with the central system of service in a case when the income from transitions between states of a network depend on states and time [13] or are random variables with the set moments of the first two orders [14]. Later in works $[10-12,15,16]$ these results extended on a case of networks of any structure. Article [16] are survey investigation methods, optimization and application of such networks when forecasting the income of various objects. Just as Markov networks with the negative and positive messages, for the first time considered by E. Gelenbe, are called G-networks, the queueing networks with the income are called HM (Howard-Matalytski) networks.

Let's consider the closed exponential queueing network with the one-type messages, consisting of $n$ QS $S_{1}, S_{2}, \ldots, S_{n}$. As states of a network we will understand a vector $k(t)=(k, t)=\left(k_{1}, k_{2}, \ldots, k_{n}, t\right)=\left(k_{1}(t), k_{2}(t), \ldots, k_{n}(t)\right)$, where $k_{i}$ - number of messages (in turn and on service) in the moment $t$ in system $S_{i}$, $i=\overline{1, n}$. The intensity of service of messages in $i$-th QS $\mu_{i}\left(k_{i}\right)$ depends on number of messages in it, $i=\overline{1, n}$. The message at transition from QS $S_{i}$ in QS $S_{j}$ brings some income, and the income of QS $S_{i}$ decreases by this size, $i, j=\overline{1, n}$. In principle, the results can be extended to a case when the message leaving QS also brings in it some income. Usually it is necessary to find the expected (average) income of systems of a network in time $t$ provided that we know its state at the initial moment $t_{0}$. Thus this task is considered in three cases when the income from transitions between states of a network is: a) the determined sizes depending on states of a network; b) the determined functions depending on states of a network and time; c) RV with the set moments of the first and second orders. It is obvious that the case a) would be a partial case of b). In the first two cases a) and b) we will assume that QS of a network are one-line, and in a case c) - multi-line.

Let's denote as $v_{i}(k, t)$ the full expected income which is gained by system $S_{i}$ in time $t$, if in an initial moment the network is in a condition $\left(k, t_{0}\right)$. The system of the difference-differential equations (DDE) for $v_{i}(k, t), i=\overline{1, n}$, for a network with disciplines of service of messages of FIFO in systems is given in systems in [16]. The number of the equations is equal in this system to the number of states of a network.

For the closed networks in case a) the system of the equations for the expected income is representable in the form of the system of final number linear non-ordinary ODE with constant coefficients for the decision of which in case of a small number 
of states of a network the method of transformations of Laplace and a direct method [15] is used.

Let's consider a closed three-level exponential HM-network, consisting of $M=n+h_{1}+\ldots+h_{n-1}$ QS, $i=1, \ldots, n, 1_{1}, \ldots, 1_{h_{1}}, \ldots,(n-1)_{1}, \ldots,(n-1)_{h_{(n-1)}}$, represented in Figure 1 which is the model of transportation of some production. In this model the system $S_{n}$ is «producer» who does production; systems $S_{1}, S_{2}, \ldots$, $S_{n-1}$ - «warehouses» in which production storage is carried out; $S_{i_{1}}, S_{i_{2}}, \ldots, S_{i_{h_{i}}}$ «points of realization of production» (shops) which arrive from a warehouse $S_{i}$, $i=\overline{1,(n-1)}$. Thus the message is understood as transportation of products of production in our logistic system «the producer - warehouses - points of realization of production».

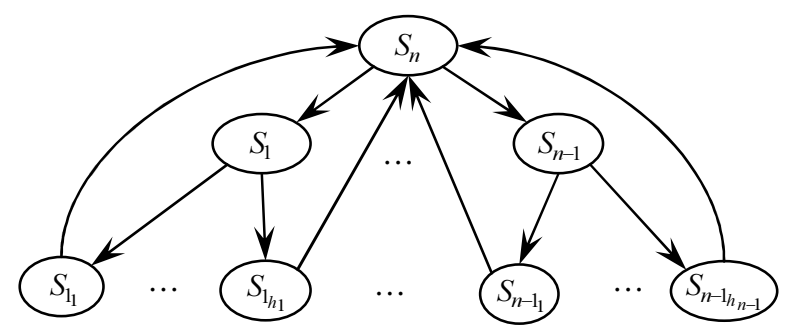

Fig. 1. Network model of transportation of products

Let's enter some sets: $X_{i}=\left\{i_{1}, i_{2}, \ldots, i_{m_{i}}\right\}, i=\overline{1,(n-1)} ; X_{0}=\bigcup_{i=1}^{n-1} X_{i} ; X=\{1$, $2, \ldots, n\} \bigcup X_{0}$. As a state of a network in a moment $t$ we will understand a vector $(k, t)=\left(k_{1}, k_{2}, \ldots, k_{n}, k_{1_{1}}, \ldots, k_{1_{h_{1}}}, \ldots, k_{(n-1)_{1}}, \ldots, k_{(n-1)_{h_{(n-1)}}}, t\right)$, where $k_{i}$ - number of states in system $S_{i}$ in a moment $t, i \in X$. The number of states of a network is equal to $L=C_{M+K-1}^{M-1}$, where $K$ - the number of states in a network. Let's consider that intervals of messages service in $S_{i}$ are distributed under the exponential law with parameter $\mu_{i}\left(k_{i}(t)\right), i=\overline{1, n}$. It means that if in a moment of time $t$ on service in $i$ - QS is $k_{i}(t)$ messages, on an interval $[t, t+\Delta t]$ service of the message ends with probability $\mu_{i}\left(k_{i}(t)\right) \Delta t+o(\Delta t), i=\overline{1, n}$.

\section{Expressions for the expected income of the central system}

Let's consider that the income from transitions between its states is the random variables (RV) with the set moments of the first two orders. Let's consider dynamics of change of the income of the central system of the $S_{n}$ "producer» network. Let's denote as $V_{n}(t)$ its income in a moment $t$. Let in an initial moment the income of 
system be equal to $V_{n}(t)=v_{n 0}$. The income of this QS in a moment $t+\Delta t$ can be submitted in a way

$$
V_{n}(t+\Delta t)=V_{n}(t)+\Delta V_{n}(t, \Delta t)
$$

where $\Delta V_{n}(t, \Delta t)$ - the change of the income of system $S_{n}$ on time interval $[t, t+\Delta t)$. In order to find this value we will write out probabilities of events which can occur during the time $\Delta t$ and changes of the income of the systems $S_{n}$, connected with these events.

1) With probability $\mu_{n}\left(k_{n}(t)\right) u\left(k_{k}(t)\right) p_{n i} \Delta t+o(\Delta t)$ the message from system $S_{n}$ will arrive in system $S_{i}$, thus the income of system $S_{i}$ will decrease by value $r_{n i}$, and the income of system $S_{n}$ will increase at this value, where $r_{n i}-\mathrm{RV}$ with expectation (exp.) $M\left\{r_{n i}\right\}=b_{n i}, i=\overline{1, n-1}$.

2) With probability $\mu_{i}\left(k_{i}(t)\right) u\left(k_{i}(t)\right) p_{i j} \Delta t+o(\Delta t)$ from system $S_{i}$ the message will arrive in $j$ QS, and its income to increase by the value $r_{i j}$ which is RV with exp. $M\left\{r_{i j}\right\}=c_{i j}, j \in X_{i}, i=\overline{1, n-1}$.

3) With probability $\mu_{i}\left(k_{i}(t)\right) u\left(k_{i}(t)\right) \Delta t+o(\Delta t)$ the message will pass from system $S_{i}$ to system $S_{n}$, thus the income $S_{i}$ will increase at a value $r_{i n}$, and the income of system $S_{n}$ will decrease by this value, where - RV with exp. $M\left\{r_{i n}\right\}=a_{i n}$, $i \in X_{j}, j=\overline{1, n-1}$.

4) With probability $1-\sum_{i, j \in X} \mu_{1}\left(k_{i}(t)\right) u\left(k_{i}(t)\right) p_{i j} \Delta t+o(\Delta t)$ on an interval of time $[t, t+\Delta t)$ change of a state of a network won't happen.

Besides, for each small period $\Delta t$ the system $S_{n}$ increases the income by value $r_{n} \Delta t$, where $r_{n}-\mathrm{RV}$ with exp. $M\left\{r_{n}\right\}=d_{n}$. Let's also consider that RV $r_{j i}$ independent in relation to $\mathrm{RV} r_{n}, i, j \in X$.

Then

$$
\Delta V_{n}(t, \Delta t)=\left\{\begin{array}{l}
r_{n i}+r_{n} \Delta t, \text { with prob. } \mu_{n}\left(k_{n}(t)\right) u\left(k_{n}(t)\right) p_{n i} \Delta t+o(\Delta t), i=\overline{1, n-1}, \\
r_{i j}+r_{n} \Delta t, \text { with prob. } \mu_{i}\left(k_{i}(t)\right) u\left(k_{i}(t)\right) p_{i j} \Delta t+o(\Delta t), j \in X_{i}, i=\overline{1, n-1}, \\
-r_{i n}+r_{n} \Delta t, \text { with prob. } \mu_{i}\left(k_{i}(t)\right) u\left(k_{i}(t)\right) \Delta t+o(\Delta t), i \in X_{i}, \\
r_{n} \Delta t, \text { with prob. } 1-\sum_{i, j \in X} \mu_{i}\left(k_{i}(t)\right) u\left(k_{i}(t)\right) p_{i j} \Delta t+o(\Delta t) .
\end{array}\right.
$$

At the fixed realization of process $k(t)$, considering (2), it is possible to write down: 


$$
\begin{aligned}
M\left\{\Delta V_{n}(\Delta t) / k(t)\right\} & =\left[\mu_{n}\left(k_{n}(t)\right) u\left(k_{n}(t)\right) \sum_{i=1}^{n-1} b_{n i} p_{n i}+\sum_{\substack{j \in X_{i} \\
i=1, n-1}} c_{i j} p_{i j} \mu_{i}\left(k_{i}(t)\right) u\left(k_{i}(t)\right)-\right. \\
& \left.-\sum_{\substack{i \in X_{j}, j=1, n-1}} a_{i n} \mu_{i}\left(k_{i}(t)\right) u\left(k_{i}(t)\right)+d_{n}\right] \Delta t+o(\Delta t)
\end{aligned}
$$

Let the system $S_{i}$ contain $m_{i}$ identical lines of service, in each of which the service time of messages is an exponentially distributed law with parameter $\mu_{i}$. Let's consider that the intensity of a service of messages in systems is line-dependent on their number, i.e. ratios are fair:

$$
\mu_{i}\left(k_{i}(t)\right)=\left\{\begin{array}{l}
\mu_{i} k_{i}(t), k_{i}(t) \leq m_{i}, \\
\mu_{i} m_{i}, k_{i}(t)>m_{i},
\end{array} \quad \mu_{i}\left(k_{i}(t)\right) u\left(k_{i}(t)\right)=\mu_{i} \min \left(k_{i}(t), m_{i}\right), i=\overline{1, n} .\right.
$$

Let's also assume that averaging of expression $\mu_{i}\left(k_{i}(t)\right) u\left(k_{i}(t)\right)$ gives, $\mu_{i} \min \left(N_{i}(t), m_{i}\right)$ i.e.

$$
M \min \left(k_{i}(t), m_{i}\right)=\min \left(N_{i}(t), m_{i}\right),
$$

where $N_{i}(t)$ - average number of demands (expecting and being served) in $S_{i}$ in a moment $t$. Taking into account this assumption we receive the following approximate ratio

$$
\begin{aligned}
M\left\{\Delta V_{n}(t, \Delta t)\right\}= & \mu_{n} \min \left(N_{n}(t), m_{n}\right) \sum_{i=1}^{n-1} b_{n i} p_{n i}+\sum_{\substack{j \in X_{i}, i=1, n-1}} c_{i j} p_{i j} \mu_{i} \min \left(N_{i}(t), m_{i}\right)- \\
& \left.-\sum_{\substack{i \in X_{j}, j=1, n-1}} a_{i n} \mu_{i} \min \left(N_{i}(t), m_{i}\right)+d_{n}\right] \Delta t+o(\Delta t) .
\end{aligned}
$$

Let's denote $v_{n}(t)=M\left\{V_{n}(t)\right\}$. From (4) we get

$$
\begin{aligned}
& v_{n}(t+\Delta t)=v_{n}(t)+M\left\{\Delta V_{n}(t, \Delta t)\right\}=v_{n}(t)+\left[\mu_{n} \min \left(N_{n}(t), m_{n}\right) \sum_{i=1}^{n-1} b_{n i} p_{n i}+\right. \\
& \left.+\sum_{\substack{j \in X_{i}, i=1, n-1}} c_{i j} p_{i j} \mu_{i} \min \left(N_{i}(t), m_{i}\right)-\sum_{\substack{i \in X_{j}, j=1, n-1}} a_{i n} \mu_{i} \min \left(N_{i}(t), m_{i}\right)+d_{n}\right] \Delta t+o(\Delta t) .
\end{aligned}
$$


Further, passing to a limit at $\Delta t \rightarrow 0$, we will receive the non-ordinary linear differential equation of the first order

$$
\begin{gathered}
\frac{d v_{n}(t)}{d t}=\mu_{n} \min \left(N_{n}(t), m_{n}\right) \sum_{i=1}^{n-1} b_{n i} p_{n i}+\sum_{\substack{j \in X_{i}, i=1, n-1}} c_{i j} p_{i j} \mu_{i} \min \left(N_{i}(t), m_{i}\right)- \\
-\sum_{\substack{i \in X_{j}, j=\overline{1, n-1}}} a_{i n} \mu_{i} \min \left(N_{i}(t), m_{i}\right)+d_{n} .
\end{gathered}
$$

Having set entry conditions $v_{n}(0)=v_{n 0}$, it is possible to find the expected income of system $S_{n}$ of a network.

The equations for the expected incomes of the second («warehouses») and the third («realization points») level can be received similarly.

\section{Technique of finding of values $N_{i}(t), i \in X$, for networks of any structure}

For finding the average number of messages in network systems in a transitional mode it is possible to use the following technique. As in a network the simplest flow of messages with an intensity $\lambda$ arrives, i.e. the probability of flow $a$ of demands in $S_{i}$ in time $\Delta t$ looks like $P_{a}(\Delta t)=\frac{\left(\lambda p_{0 i} \Delta t\right)^{a}}{a !} e^{-\lambda p_{0 i} \Delta t}, a=0,1,2, \ldots$, the average number of the messages which have arrived from the outside in $S_{i}$ in time $\Delta t$ is equal to $\lambda p_{0 i} \Delta t$. Let's denote as $\bar{\rho}_{i}(t)$ - the average number of busy lines of service in $S_{i}$ in a moment $t, i=\overline{1, n}$. Then $\mu_{i} \bar{\rho}_{i}(t) \Delta t$ - average number of the messages which have left $S_{i}$ in time $\Delta t$, and $\sum_{\substack{j=1 \\ j \neq i}}^{n} \mu_{j} \rho_{j}(t) p_{j i} \Delta t$ - average number of the messages which have arrived in $S_{i}$ from other subjects in time $\Delta t$. Therefore

$$
N_{i}(t+\Delta t)-N_{i}(t)=\lambda p_{0 i} \Delta t+\sum_{\substack{j=1 \\ j \neq i}}^{n} \mu_{j} \rho_{j}(t) p_{j i} \Delta t-\mu_{i} \rho_{i}(t) \Delta t, i=\overline{1, n},
$$

and if $\Delta t \rightarrow 0$ the system of ODE for $N_{i}(t)$ flows:

$$
\frac{d N_{i}(t)}{d t}=\sum_{\substack{j=1 \\ j \neq i}}^{n} \mu_{j} \bar{\rho}_{j}(t) p_{j i}-\mu_{i} \bar{\rho}_{i}(t)+\lambda p_{0 i}, i=\overline{1, n}
$$


The value $\bar{\rho}_{i}(t)$ can be approximated by the expression $\bar{\rho}_{i}(t)=$ $=\left\{\begin{array}{l}N_{i}(t), N_{i}(t) \leq m_{i}, \\ m_{i}, N_{i}(t)>m_{i},\end{array}=\min \left(N_{i}(t), m_{i}\right)\right.$. Then the system of equations (6) will look like

$$
\frac{d N_{i}(t)}{d t}=\sum_{\substack{j=1 \\ j \neq i}}^{n} \mu_{j} \min \left(N_{j}(t), m_{j}\right) p_{j i}-\mu_{i} \min \left(N_{i}(t), m_{i}\right)+\lambda p_{0 i}, i=\overline{1, n} .
$$

It is a system of the linear non-ordinary ODE with explosive right parts. It is necessary to solve it by splitting the phase space into a number of areas and finding the decision in each of them.

If the network is closed and functions so that on the average queues are not observed in it, i.e. $\min \left(N_{i}(t), m_{i}\right)=N_{i}(t), i=\overline{1, n}$, the system (7) will look like:

$$
\frac{d N_{i}(t)}{d t}=\sum_{\substack{j=1 \\ j \neq i}}^{n} \mu_{j} p_{j i} N_{j}(t)-\mu_{i} N_{i}(t), i=\overline{1, n}
$$

It can be written down in a matrix form $\frac{d N(t)}{d t}=Q N(t)$, where $N^{T}(t)=\left(N_{1}(t), N_{2}(t), \ldots, N_{n}(t)\right), Q$ - the square matrix consisting of elements $q_{i j}=\mu_{j} p_{j i}$ if we put $p_{i i}=-1, i, j=\overline{1, n}$. The decision of the last system looks like $N(t)=N(0) e^{Q t}$, where $N(0)$ - some set entry conditions. For our closed three-level network system of the equations (8) becomes

$$
\left\{\begin{array}{l}
\frac{d N_{i}(t)}{d t}=\mu_{n} N_{n}(t) p_{n i}-\mu_{i} N_{i}(t), \\
\frac{d N_{j}(t)}{d t}=\sum_{i=1}^{n-1} \mu_{i} N_{i}(t) p_{i j}-\mu_{j} N_{j}(t), j \in X_{i}, i=\overline{1, n-1} . \\
\frac{d N_{n}(t)}{d t}=\sum_{\substack{j \in X_{i} \\
i=1, n-1}} \mu_{j} N_{j}(t)-\mu_{n} N_{n}(t),
\end{array}\right.
$$

Example. Let's consider the closed queueing network with $n=7$ QS, Figure 1, systems of the third bottom level denote as $S_{4}, S_{5}, S_{6}, S_{7}$. Let's consider network functioning on time interval $[0 ; 15]$. Let intensity of service of messages not depend on their number: probabilities of transitions of messages between $\mathrm{QN}$ of a network are equal to $p_{1 i}=0.5, i=4,5, p_{2 i}=0.5, i=6,7, p_{3 i}=0.5, i=1,2, p_{i 3}=1$, $i=4,5,6,7, p_{i j}=0, i, j=\overline{1,7}$, and $K=20, m_{i}=2, i=1,3,4, m_{i}=3, i=2,5,6$, 
$m_{7}=8, a_{14}=14, a_{15}=12, a_{26}=13, a_{27}=16, b_{43}=9, b_{53}=b_{73}=8, b_{63}=10$, $c_{31}=25, c_{32}=29, d_{1}=d_{7}=2, d_{2}=3, d_{3}=4, d_{4}=1, d_{5}=5, d_{6}=7$. In this example $N_{i}(0)$ we assume: $N_{i}(0)=2, i=1,6, N_{2}(0)=5, N_{3}(0)=4, N_{4}(0)=3$, $N_{5}(0)=3, N_{7}(0)=1$. Then, using (9), it is possible to find values for the average number of messages $N_{i}(t)$, and then expressions for the expected income of systems $S_{i}, i=\overline{1, n}$. The program in a Mathematica package was created for this purpose, schedules of these values are given in Figure 2.

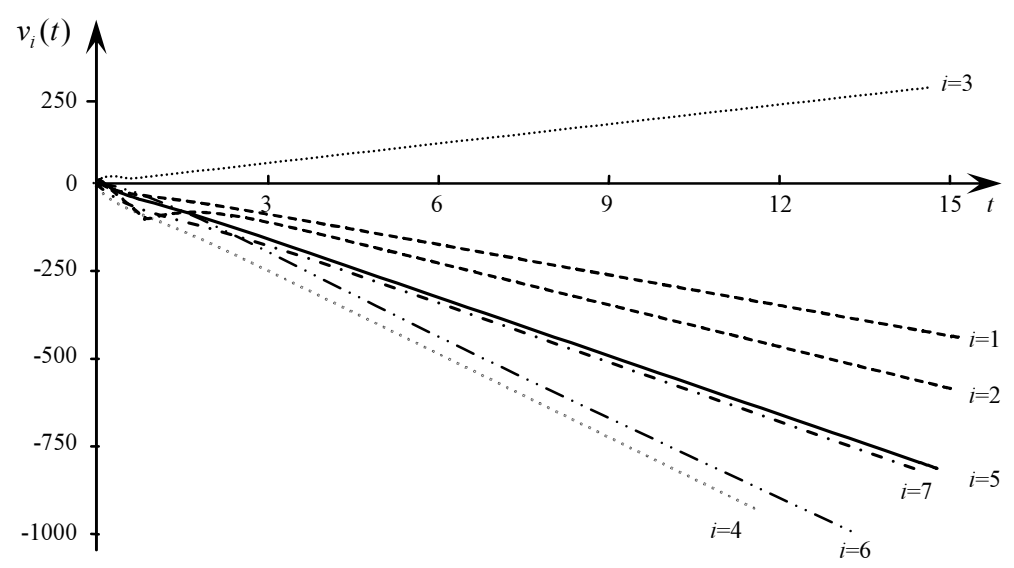

Fig. 2. Expected incomes of network systems

\section{Finding of variances of the income of the central system}

For the expected income of the central QS, as follows from (5), the following expression is fair:

$$
\begin{gathered}
v_{n}(t)=v_{n 0}+d_{n} t+\mu_{n} \int_{0}^{t} \min \left(N_{n}(s), m_{n}\right) d s \sum_{i=1}^{n-1} b_{n i} p_{n i}- \\
-\sum_{\substack{i \in X_{j}, j=1, n-1}} a_{i n} \mu_{i} \int_{0}^{t} \min \left(N_{i}(s), m_{i}\right) d s+\sum_{\substack{j \in X_{i}, i=1, n-1}} c_{i j} p_{i j} \mu_{i} \int_{0}^{t} \min \left(N_{i}(s), m_{i}\right) d s .
\end{gathered}
$$

Let's denote

$$
\begin{gathered}
M\left\{r_{j n}{ }^{2}\right\}=a_{2 j n}, M\left\{r_{n i}{ }^{2}\right\}=b_{2 n i}, M\left\{r_{i j}{ }^{2}\right\}=c_{2 i j}, j \in X_{i}, i=\overline{1, n-1}, \\
M\left\{r_{n}^{2}\right\}=d_{2 n} .
\end{gathered}
$$


Let's consider difference square $\left(V_{n}(t)-v_{n 0}\right)^{2}$ :

$$
\begin{gathered}
\left(V_{n}(t)-v_{n 0}\right)^{2}=\left(v_{n 0}+\sum_{l=1}^{m} \Delta V_{n l}(t)-v_{n 0}\right)^{2}=\left(\sum_{l=1}^{m} \Delta V_{n l}(t)\right)^{2}= \\
=\sum_{l=1}^{m} \Delta V_{n l}^{2}(t)+\sum_{\substack{l=1 \\
l}}^{m} \sum_{\substack{j \neq 1 \\
l \neq j}}^{m} \Delta V_{n l}(t) \Delta V_{n j}(t) .
\end{gathered}
$$

Let's find exp. of composed in the right part of the last equality. For this purpose we will write down auxiliary equalities, considering that $\mathrm{RV} r_{j i}$ are in pairs independent from $r_{i}, i, j \in X$. Then

$$
\begin{gathered}
M\left\{\left(-r_{j n}+r_{n} \Delta t\right)^{2}\right\}=a_{2 j n}-2 a_{j n} d_{n} \Delta t+d_{2 n}(\Delta t)^{2}, \\
M\left\{\left(r_{n i}+r_{n} \Delta t\right)^{2}\right\}=b_{2 n i}+2 b_{n i} d_{n} \Delta t+d_{2 n}(\Delta t)^{2}, \\
M\left\{\left(r_{i j}+r_{n} \Delta t\right)^{2}\right\}=c_{2 i j}+2 c_{i j} d_{n} \Delta t+d_{2 n}(\Delta t)^{2} .
\end{gathered}
$$

Considering (12)-(14), we get:

$$
\begin{gathered}
M\left\{\Delta V_{n l}^{2}(t) / k(t)\right\}=\sum_{\substack{j \in X_{i}, i=1, n-1}}\left[a_{2 j n}-2 a_{j n} d_{n} \Delta t\right] \mu_{j}\left(k_{j}(l)\right) u\left(k_{j}(l)\right) \Delta t+ \\
+\sum_{i=1}^{n-1}\left[b_{2 n i}+2 b_{n i} d_{n} \Delta t\right] \mu_{n}\left(k_{n}(l)\right) u\left(k_{n}(l)\right) p_{n i} \Delta t+ \\
+\sum_{\substack{j \in X_{i}, i=1, n-1}}\left[c_{2 i j}+2 c_{i j} d_{n} \Delta t\right] \mu_{i}\left(k_{i}(l)\right) u\left(k_{i}(l)\right) p_{i j} \Delta t+o(\Delta t) .
\end{gathered}
$$

Considering that at the fixed realization of $k(t)$ values $\Delta V_{n l}(t)$ and $\Delta V_{n j}(t)$ are independent at $l \neq j$, it is possible to find

$$
\begin{gathered}
M\left\{\Delta V_{n l}(t) \Delta V_{n j}(t) / k(t)\right\}=\left\{d_{n}+\mu_{n}\left(k_{n}\right) u\left(k_{n}(t)\right) \sum_{i=1}^{n-1} b_{n i} p_{n i}-\right. \\
\left.\left.-\sum_{\substack{i \in X_{j}, j=1, n-1}} a_{i n} \mu_{i}\left(k_{i}(t)\right) u\left(k_{i}(t)\right)+\sum_{\substack{j \in X_{i}, i=1, n-1}} c_{i j} p_{i j} \mu_{i}\left(k_{i}(t)\right) u\left(k_{i}(t)\right)\right] \Delta t+o(\Delta t)\right\}^{2}=o(\Delta t) .
\end{gathered}
$$


Then, doing limit transition at $\Delta t \rightarrow 0$, from (10), (15), (16) and that $m \Delta t=t$, we receive

$$
\begin{gathered}
M\left\{\left(V_{n}(t)-v_{n 0}\right)^{2} / k(t)\right\}=\sum_{l=1}^{m} M\left\{\Delta V_{n l}^{2}(\Delta t) / k(t)\right\}+ \\
+\sum_{\substack{l=1 \\
l}}^{m} \sum_{\substack{q=1 \\
q \neq l}}^{m}\left\{\Delta V_{n l}(\Delta t) \Delta V_{n q}(\Delta t) / k(t)\right\}= \\
=\sum_{\substack{j \in X_{i}, i=1, n-1}} a_{2 j n} \mu_{j} \int_{0}^{t} \min \left(k_{j}(s), m_{j}\right) d s+\sum_{i=1}^{n-1} b_{2 n i} \mu_{n} p_{n i} \int_{0}^{t} \min \left(k_{n}(s), m_{n}\right) d s+ \\
+\sum_{\substack{j \in X_{i}, i=1, n-1}} c_{2 i j} \mu_{j} p_{i j} \int_{0}^{t} \min \left(k_{i}(s), m_{i}\right) d s .
\end{gathered}
$$

Averaging that expression by $k(t)$ we get

$$
\begin{gathered}
M\left\{\left(V_{n}(t)-v_{n 0}\right)^{2}\right\}=\sum_{\substack{j \in X_{i}, i=1, n-1}} \mu_{j} a_{2 j n} \int_{0}^{t} \min \left(N_{j}(s), m_{j}\right) d s+ \\
+\sum_{i=1}^{n-1} b_{2 n i} \mu_{n} p_{n i} \int_{0}^{t} \min \left(N_{n}(s), m_{n}\right) d s+\sum_{\substack{j \in X_{i}, i=1, n-1}} c_{2 i j} \mu_{j} p_{i j} \int_{0}^{t} \min \left(N_{i}(s), m_{i}\right) d s .
\end{gathered}
$$

Let's find expression for $M^{2}\left\{\left(V_{n}(t)-v_{n 0}\right)\right\}$, using (10):

$$
\begin{gathered}
M^{2}\left\{\left(V_{n}(t)-v_{n 0}\right)\right\}=\left\{d_{n} t+\mu_{n} \int_{0}^{t} \min \left(N_{n}(s), m_{n}\right) d s \sum_{i=1}^{n-1} b_{n i} p_{n i}-\right. \\
\left.-\sum_{\substack{i \in X_{j}, j=1, n-1}} a_{i n} \mu_{i} \int_{0}^{t} \min \left(N_{i}(s), m_{i}\right) d s+\sum_{\substack{j \in X_{i}, i=1, n-1}} c_{i j} p_{i j} \mu_{i} \int_{0}^{t} \min \left(N_{i}(s), m_{i}\right) d s\right\} .
\end{gathered}
$$

Thus, the variance of the income of the central QS of a network taking into account the last two ratios, it is possible to write it down in a way

$$
D V_{n}(t)=D\left\{V_{n}(t)-v_{n 0}\right\}=M\left\{\left(V_{n}(t)-v_{n 0}\right)^{2}\right\}-M^{2}\left\{\left(V_{n}(t)-v_{n 0}\right)\right\},
$$

variances of the incomes of systems of the second level and the third level can be found similarly, as well as for the central system. 


\section{Conclusions}

The main results can be the following:

1. It is considered the closed three-level HM queueing network, which is a model of the production transportation in LTS.

2. An approximate relation for the expected income of the central system in the case when the income from transitions between conditions of a network with multi-line QS is RV with the set moments of the first two orders and system nonhomogeneous differential equations for an average number of messages in network systems are received. The example of finding the income was given.

3. An expression for variances of the income of the central queueing system was also received.

4. The above characteristics for the second- and the third-level network can be found similarly.

Further investigations will be connected with the investigation and HM-networks optimization with arbitrary distributions of the service times of the messages in the systems and to their application when forecasting the incomes of LTS.

\section{References}

[1] Brodetski L., Special modifications of criterion of a choice for optimization of chains of deliveries in the conditions of uncertainty, Logistics Today 2009, 3, 156-167 (in Russian).

[2] Brodetski L., How to order orders in chains of deliveries taking into account decrease in tariffs of penalties, Logistics Today 2011, 3, 148-155 (in Russian).

[3] Brodetski L., Tokarev E., Invariant to decrease of tariffs of penalties of model of optimization of an order of implementation of orders in chains of deliveries, Part II, Logistics 2012, 11, 50-54 (in Russian).

[4] Guillen G., Mele F., Bagajewicz M., Espuna A., Puigjaner L., Multiobjective supply chain design under uncertainty, Chem. Eng. Sciences 2005, 6, 1535-1553.

[5] Nerush Y., Commercial Logistics, Banks and Exchanges: YuNITI, Moscow 1997, 272 p. (in Russian).

[6] Oleynik A.V., Use of mathematical apparatus of the theory of mass service in logistic system of cross-border economic cooperation of the western region of Belarus, Logistics: current trends of development, II International Scientific and Practical Conference, St. Petersburg, 10-11 Apr. 2003, Theses of reports, 144-148 (in Russian).

[7] Howard R., Dynamic Programming and Markov Processes, Soviet Radio, Moscow 1964, 110 p. (in Russian).

[8] Matalytski M., Pankov A., Incomes probabilistic models of the banking network, Scientific Research of the Institute of Mathematics and Computer Science of Czestochowa University of Technology 2003, 1, 2, 99-104.

[9] Matalytski M., Pankov A., About forecasting of the income in the closed Markov network with the central system, The Review of Applied and Industrial Mathematics 2006, 13, 1, 123-125 (in Russian).

[10] Matalytski M., Tikhonenko O., Koluzayeva E., Systems and Networks of Mass Service: Analysis and Application, Monograph, GRGU, Grodno 2011, 818 p. (in Russian). 
[11] Matalytski M., Koluzaeva E., Analysis and optimization of Markov HM-networks with stochastic incomes from transitions between their states, Scientific Research of the Institute of Mathematics and Computer Science of Czestochowa University of Technology 2008, 1, 7, 22-27.

[12] Matalytski M., Koluzaeva E., Finding of expected incomes in open exponential networks of arbitrary architecture, Scientific Research of the Institute of Mathematics and Computer Science of Czestochowa University of Technology 2007, 1, 6, 179-190.

[13] Matalytski M., Pankov A., Investigation of incomes in Markov network with central system, Computer Science 2005, 5, 8, 7-17.

[14] Pankov A.V., The analysis of the expected income in the closed stochastic network with the central system, Vestnik GrGU, Series 2, Mathematics, Physics, Informatics, Computer Facilities and Management 2006, 2, 48-56 (in Russian).

[15] Matalytski M.A., Koluzayeva E.V., Markov networks of mass service of any topology with the income, Reports of NAN RB 2009, 53, 3, 10-17 (in Russian).

[16] Matalytski M.A., About some results of the analysis and optimization of Markov networks with the income and their application, Automatic Equipment and Telemechanics 2009, 10, 97-113 (in Russian). 
